\title{
Contralateral radiculopathy after transforaminal lumbar interbody fusion
}

\author{
Travis Hunt • Francis H. Shen · Christopher I. Shaffrey • \\ Vincent Arlet
}

Received: 4 April 2006/Revised: 22 January 2007/Accepted: 29 March 2007/Published online: 9 May 2007

(C) Springer-Verlag 2007

\begin{abstract}
Transforaminal lumbar interbody fusion (TLIF) is an effective treatment for patients with degenerative spondylolisthesis and degenerative disc disease. Opposite side radiculopathy after the TLIF procedure has been recognized in this institution but has not been addressed in the literature. We present a case of opposite side radiculopathy after the TLIF procedure. We believe that this complication is related to asymptomatic stenosis on the contralateral side that is unmasked by the increased lordosis of the TLIF. The authors recommend increasing both disc height and foraminal height when choosing an interbody graft, and possibly decompressing the opposite foramen when preoperative MRI demonstrates foraminal stenosis.
\end{abstract}

Keywords TLIF · Complication · Spondylolisthesis · Radiculopathy

\section{Background}

Transforaminal lumbar interbody fusion (TLIF) is an effective treatment for patients with degenerative spond-

Nothing was received from industry to support this paper. An reviewer's comment on this paper is available at http://dx.doi.org/ 10.1007/s00586-007-0389-8.

T. Hunt $\cdot$ F. H. Shen $\cdot$ V. Arlet $(\bowtie)$

Department of Orthopaedic Surgery, University of Virginia,

PO Box 800159, Charlottesville, VA 22908-0159, USA

e-mail: VA3E@hscmail.mcc.virginia.edu

C. I. Shaffrey

Department of Neurosurgery, University of Virginia,

PO Box 800159, Charlottesville, VA 22908-0159, USA ylolisthesis and degenerative disc disease [4]. High success rates and few complications have been reported. The TLIF technique has comparable results to other interbody fusions, such as posterolumbar interbody fusion (PLIF) and anterior lumbar interbody fusion (ALIF) techniques. The potential advantages of the TLIF technique over the ALIF include avoidance of the anterior approach and its related complications. Advantages of the TLIF over the PLIF are the need of less retraction of the nerve roots and cauda equina as the facets on one side are totally resected and the approach to the disc space is further lateral to the PLIF where the facets are theoretically resected [3]. Classic technique for TLIF surgery is usually done on the side of the symptomatic radiculopathy, and the contralateral facets and foramina are left intact [2,5]. Decompression of the opposite side has not been judged necessary in the past as it has been commonly assumed that the TLIF spacer increases the disc space height and the foraminal height in the opposite foramen of the TLIF approach. The authors believe that this is an incorrect assumption. Opposite side radiculopathy after the TLIF procedure has been recognized in this institution but has not been addressed in the literature.

This complication has been recognized six times in the last 18 months by four separate experienced surgeons representing an incidence rate of $2.5 \%$. We present one such case for illustration.

\section{Case example}

A 65-year-old healthy woman complained of back pain and right leg pain after a previous discectomy 18 months earlier. She had no neurological deficits. X-rays demonstrated a degenerative L4-5 spondylolisthesis (Figs. 1, 2). 
Fig. 1 Preoperative X-rays demonstrate degenerative spondylolisthesis in a 65 -yearold with right leg pain
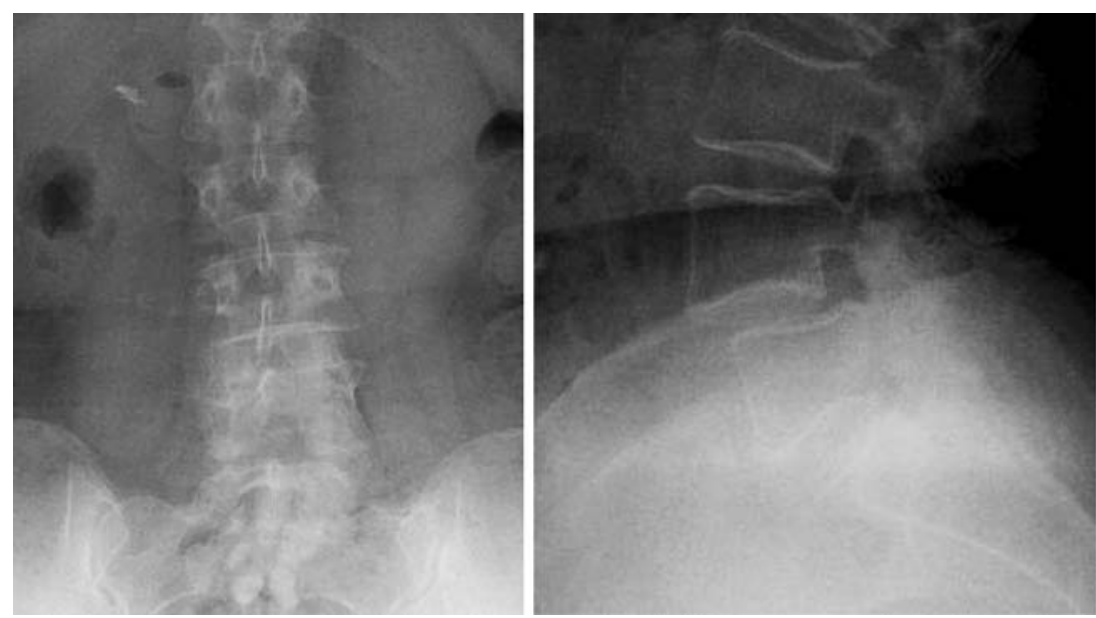

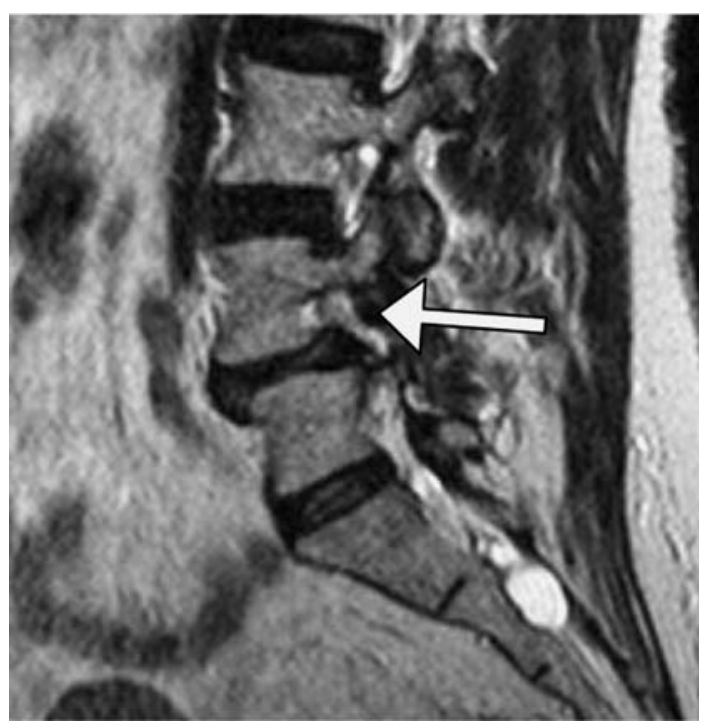

Fig. 2 Preoperative mri demonstrates right L4-5 foraminal stenosis

After failed conservative treatment, the patient underwent a right sided TLIF of L4-5 with decompression of the right side. The dura was protected as the disc space was prepared and the spacer was inserted. No immediate complications were noted.

Post-operatively, while still in the hospital, the patient reported no right leg pain, however, complained of left leg pain in L4 distribution. Post operative X-rays and CT scan showed no obvious malpositioning of the instrumentation or disc material compressing the nerve, but did demonstrate foraminal stenosis on the left side. The patient underwent a series of three epidural steroid injections which alleviated the left leg symptoms. The patient is now doing well with no leg or back symptoms. Review of the initial MRI demonstrated that although stenosis was worse on the right side, it was existent on the left (Fig. 3).

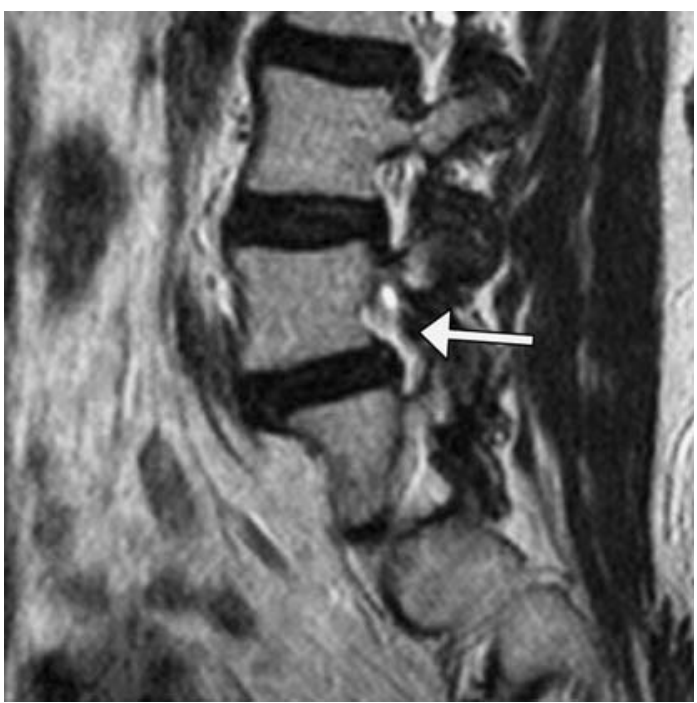

Fig. 3 Preoperative MRI reveals asymptomatic left foraminal L4-5 stenosis

\section{Discussion}

TLIF is an effective treatment for degenerative disease of the spine and facilitates effective $360^{\circ}$ fusion. Reported complications include ipsilateral nerve injury, medical complications, wound infections, continued pain, and cerebrospinal fluid leak. Opposite side radiculopathy is a complication which may be under recognized. A contralateral disc herniation after TLIF was reported in the European literature, however, it was unclear in that situation whether increasing lordosis contributed to the disc herniation in that particular situation [1] (Figs. 4, 5, 6).

We present a case of a patient who complained of contralateral leg pain after the TLIF procedure. Postoperative radiography revealed no implant malpositioning. 
Fig. 4 Postoperative TLIF from the right side. The patient developed new onset left leg pain in L4 distribution
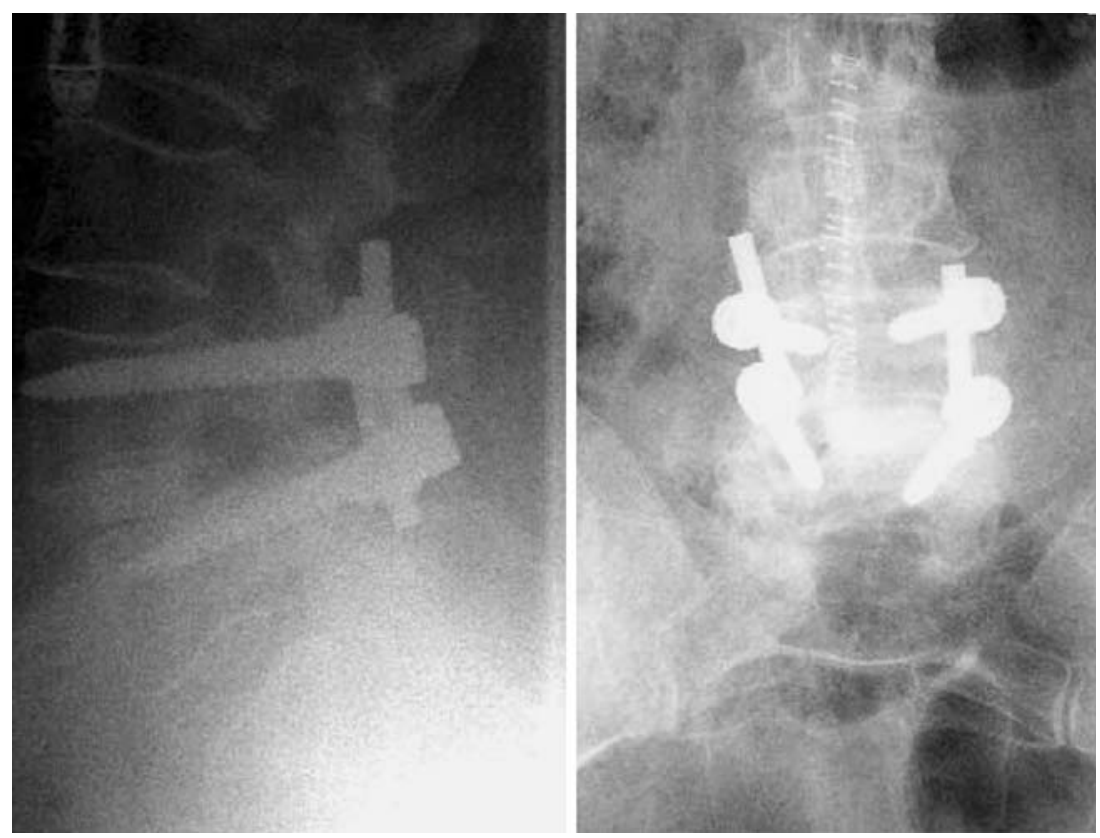

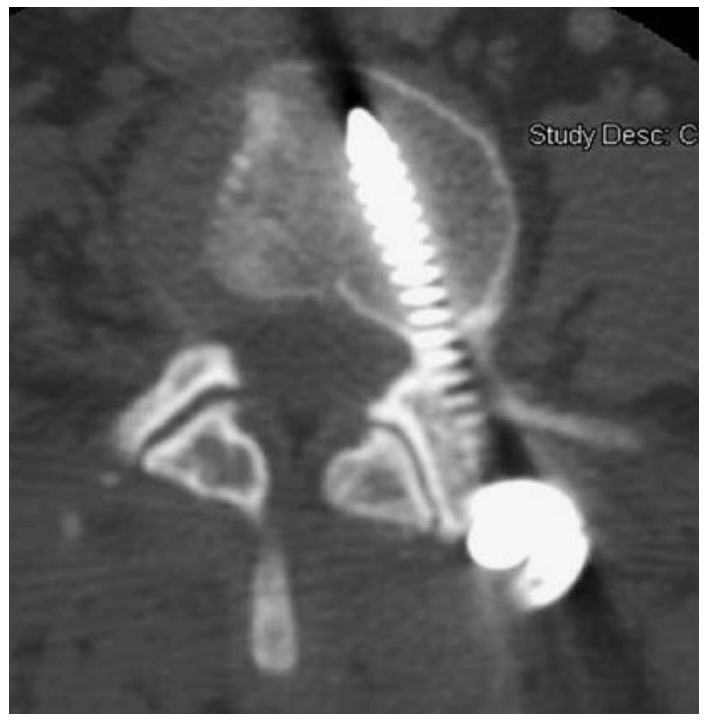

Fig. 5 The post operative CT scan revealed that the left pedicle screw was clearly outside the spinal canal and not responsible for the post operative leg pain

The authors believe that the injury to the contralateral nerve root is a result of several different scenarios:

- During the TLIF procedure, the distraction on the pedicle screws inserted on the side of the TLIF open the disc space on the TLIF side but may wedge the opposite foramen and induce compression at this level.

- The necessary distraction to achieve restoration of the disc space height with the different trials wedges the disc space on the opposite side during the initial phase of the insertion.

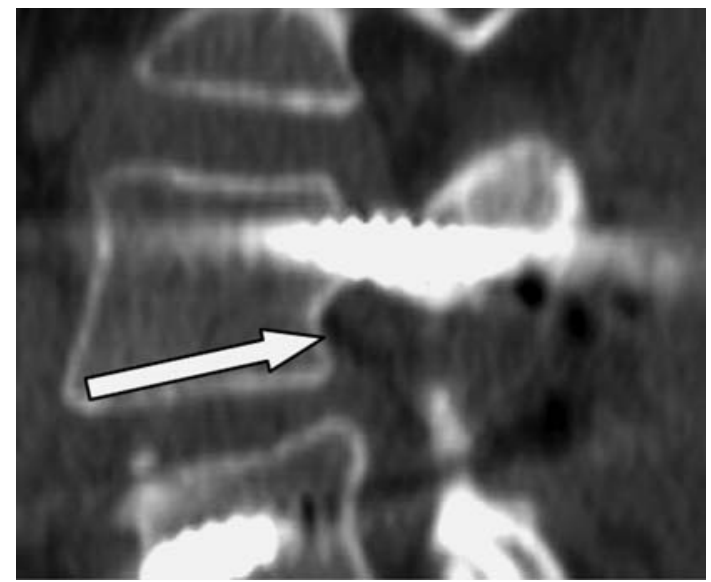

Fig. 6 The postoperative CT revealed stenosis in the left foramen

- The increased and desirable lordosis achieved through a TLIF procedure may result in further foraminal stenosis on the non decompressed side.

Careful preoperative planning is important when deciding the height of the interbody graft. Restoring normal lordosis in a hypolordotic degenerative spine can unmask stenosis. It is often incorrectly assumed by the surgeon that insertion of a graft which increases disk height concomitantly increases foraminal height. This can be seen in the case example where the disk height was increased, but the foraminal height was decreased (Fig. 7).

Insertion of the spacer as anterior as possible into the vertebral space increases lordosis but closes down the posterior elements and potentiates stenosis. The authors recommend careful review of the preoperative $\mathrm{CT}$ scans 


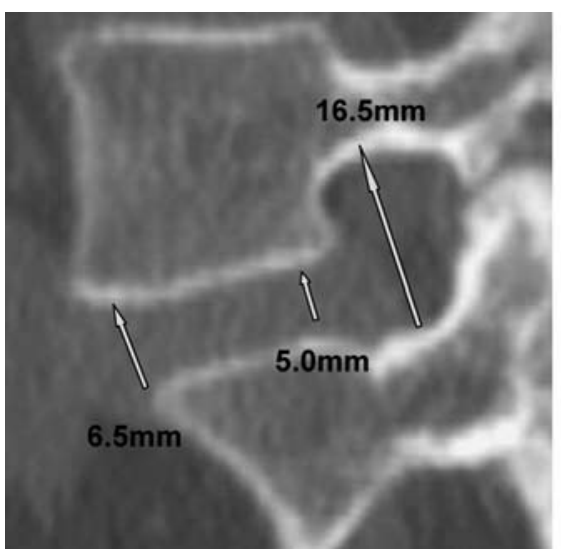

Fig. 7 Preoperative and postoperative CT scans after the TLIF procedure. The TLIF graft is not appreciated as the cut is at the level of the foramen. The CT scans are different in scale, but are measured with a computer imaging tool in order to give accurate measurements.

and MRI paying attention to the opposite side when deciding whether to perform opposite side foraminotomy during the TLIF. The use of distraction across a rod on the contralateral side of the interbody insertion may protect the opposite side foramen when performing the diskectomy and insertion of the anterior interbody graft.

The use of a lamina spreader on pedicle screws when inserting grafts worsens stenosis on the contralateral side by wedging the foramen. We recommend use of the lamina spreader on the spinous processes when it is possible to lessen potential opposite side compression. We also recommend checking the opposite foramen with a curved ball probe after insertion of the graft and restoring lordosis if the height of the posterior spine is not increased. Anatomic reduction of a degenerative spondylolisthesis may lessen the chance of opposite side radiculopathy, and the use of electromyography and nerve conduction studies may be beneficial.

\section{Conclusion}

Opposite side radiculopathy is a potential complication in TLIF surgery which can be avoided. Predicting which patients would benefit from bilateral decompression in asymptomatic patients is difficult. If the chosen side of TLIF is dictated by the patients' symptoms, surgeons should

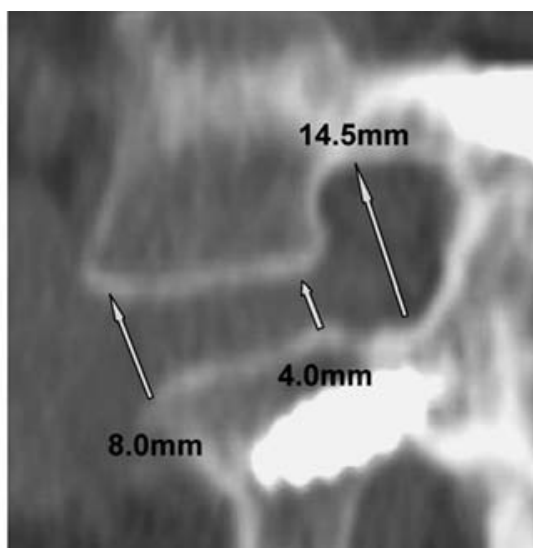

Although the anterior disc space height has been increased from 6.5 to $8.0 \mathrm{~mm}$, the posterior disc height has been decreased from $5.0 \mathrm{~mm}$ to $4.0 \mathrm{~mm}$. The foraminal height has decreased due to the increased lordosis

look at the opposite foramen when planning the TLIF procedure to see if there is preexistent stenosis. In these cases where stenosis exists, the opposite side should be decompressed. This potential complication must be recognized by the treating surgeon when it occurs as prompt treatment and can avoid possible long term impairment. Patients should be warned about this potential complication in presurgical discussions.

\section{References}

1. Hackenberg L, Halm H, Bullmann V, Vieth V, Schneider M, Liljenqvist U (2005) Transforaminal lumbar interbody fusion: a safe technique with satisfactory three to five year results. Eur Spine J 14(6):551-558

2. Harms JG, Jeszenszky D (1998) The unilateral, transforaminal approach for posterior lumbar interbody fusion. Orthop Traumatol 6:88-99

3. Humphreys SC, Hodges SD, Patwardhan AG, Eck JC, Murphy RB, Covington LA (2001) Comparison of posterior and transforaminal approaches to lumbar interbody fusion. Spine 26(5):567-571

4. Potter BK, Freedman BA, Verwiebe EG, Hall JM, Polly DW Jr, Kuklo TR (2005) Transforaminal lumbar interbody fusion: clinical and radiographic results and complications in 100 consecutive patients. J Spinal Disord Tech 18(4):337-346

5. Salehi SA, Tawk R, Ganju A, LaMarca F, Liu JC, Ondra SL (2004) Transforaminal lumbar interbody fusion: surgical technique and results in 24 patients. Neurosurgery 54(2):368-374 